\title{
Discriminação de incrementos de desflorestamento na Amazônia com dados SAR R99B em banda L
}

\author{
Júlio Bandeira GUERRA ${ }^{1}$ José Claudio MURA², Corina da Costa FREITAS³
}

\begin{abstract}
RESUMO
O uso de dados de sensoriamento remoto óptico em projetos de monitoramento de extensas áreas de floresta tropical é limitado devido à intensa cobertura por nuvens. Os dados SAR (Synthetic Aperture Radar) podem ser uma alternativa interessante para detectar desflorestamento nas regióes de floresta tropical onde a cobertura por nuvens é permanente. Neste contexto, o objetivo deste trabalho é avaliar o potencial do dado SAR adquirido em banda L pelo sistema aerotransportado R99B da Força Aérea Brasileira (FAB) para discriminar incremento de desflorestamento na Amazônia. Para tanto, foram realizadas classificaçóes MAXVER-ICM com dados SAR multipolarizados de uma área teste localizada na região Sudeste do Estado do Acre. As classificaçôes realizadas com a combinação dos canais $\mathrm{HH}, \mathrm{HV}$ e VV e com o par de polarizações $\mathrm{HH}+\mathrm{HV}$ obtiveram boa concordância com o mapa produzido no projeto PRODES ( $\mathrm{k}=0,68$, onde $\mathrm{k}$ é o índice Kappa), o qual foi adotado como dado de referência. Este resultado indica que o dado SAR multipolarizado em banda L possui bom potencial para discriminar incremento de desflorestamento na Amazônia.
\end{abstract}

PALAVRAS-CHAVE: SAR R99B, banda L, multipolarização, desflorestamento. SAR R99B, L band, multipolarization, desforestation.

\section{Discriminating deforestation increment areas in the Amazon rainforest with L band SAR R99B data}

\begin{abstract}
The use of optical remote sensing data in large tropical forest regions has an important limitation due to cloud cover. Synthetic Aperture Radar (SAR) data can be a viable alternative in areas where cloud cover is permanent, because the data acquisition is independent on atmospheric conditions. In this context, the main objective of this work was to evaluate the potential of $\mathrm{L}$ band SAR data acquired by R99B Brazilian Air Force (FAB) airborne system to discriminate deforestation increments in the Amazon rainforest. In order to achieve this purpose, we performed Maximum Likelihood classifications with multipolarized SAR data of a test site located in the state of Acre. The classifications performed with the combination of three channels $(\mathrm{HH}+\mathrm{HV}+\mathrm{VV})$ and with the polarization pair $\mathrm{HH}+\mathrm{HV}$ obtained good agreement with PRODES reference map $(\mathrm{k}=0,68$, where $\mathrm{k}$ is de Kappa index). This result indicates that multipolarized L band SAR data have good potential to discriminate deforestation increments in the Amazon rainforest.
\end{abstract}

KEYWORDS: SAR R99B, L band, multipolarization, deforestation.

${ }_{1}$ Instituto Nacional de Pesquisas Espaciais (INPE) - E-mail: guerra@dsrinpe.br

${ }^{2}$ Instituto Nacional de Pesquisas Espaciais (INPE) - E-mail: mura@dpi.inpe.br

${ }^{3}$ Instituto Nacional de Pesquisas Espaciais (INPE) -E-mail: corina@dpi.inpe.br 


\section{INTRODUÇÃO}

O acompanhamento sistemático do desflorestamento da Amazônia Brasileira é realizado pelos projetos PRODES (Projeto de Monitoramento do Desflorestamento da Amazônia Legal) (Câmara et al., 2006) e DETER (Sistema de Detecçấo de Desflorestamento em Tempo Real) (INPE, 2008), ambos desenvolvidos pelo Instituto Nacional de Pesquisas Espaciais (INPE). O projeto PRODES tem o objetivo de mapear e estimar anualmente a taxa de desflorestamento da Amazônia Brasileira com base em imagens TM/Landsat, CCD/CBERS e DMC. No projeto DETER é realizada a detecçáo de áreas desflorestadas por corte raso e de áreas em processo de degradaçáo florestal progressiva a partir de imagens MODIS/ TERRA e WFI/CBERS. O DETER emite alertas mensais de desflorestamento para subsidiar açóes de fiscalização.

Entretanto, o uso de dados de sensores remotos ópticos em extensas áreas de floresta tropical tem uma forte limitação devido à intensa cobertura por nuvens. Em algumas regióes da Floresta Amazônica, a probabilidade de obtenção de imagens TM/Landsat (ou de outro sistema óptico similar) com mínima cobertura por nuvens é pequena durante quase todo o ano (Asner, 2001).

As imagens SAR podem ser uma alternativa interessante para detectar desflorestamento em floresta tropical, uma vez que o sinal de microondas penetra as nuvens, o que torna possível a aquisição de dados independentemente das condiçōes atmosféricas. Além disso, os dados SAR são sensíveis às variaçóes de estrutura/biomassa da floresta, fato que permite a discriminação de diferentes classes de cobertura do solo em ambiente florestal.

Diversos trabalhos evidenciaram o potencial de dados SAR para aplicaçôes em floresta tropical (Santos et al. 2003; Sgrenzaroli et al., 2004, Kuplich, 2006, Freitas et al., 2008). Porém, em alguns casos, dados SAR monopolarizados apresentaram limitaçóes para detectar novos desflorestamentos por corte raso (Bufalo, 2005), especialmente áreas recém desflorestadas (Almeida Filho et al., 2005) (áreas onde o processo de desflorestamento por corte raso não foi concluído, ou seja, troncos e galhos ainda permanecem sobre o solo após a derrubada e queima das árvores).

Os dados adquiridos por sensores SAR polarimétricos guardam mais informação sobre a interação do sinal transmitido com o alvo que os dados adquiridos por sistemas SAR monopolarizados convencionais. Este acréscimo de informação pode significar um avanço na discriminação de classes de uso e cobertura do solo e na detecção de mudança de cobertura em floresta tropical. Em Hoekman \& Quiñones (2000), a classificação MAXVER-ICM do dado AIRSAR em banda $\mathrm{L}$ e polarizações $\mathrm{HH}+\mathrm{HV}+\mathrm{VV}$ obteve confusão entre as classes de floresta e desflorestamento recente aproximadamente
$80 \%$ inferior à obtida na classificaçáo realizada somente com o canal $\mathrm{HH}$.

O sistema SAR aerotransportado R99B foi concebido no âmbito do projeto SIVAM (Sistema de Vigilância da Amazônia) para realizar imageamentos na Amazônia Legal. Os dados adquiridos por este sensor, que opera no modo polarimétrico em banda L e interferométrico em banda X, podem apresentar bom potencial para detectar e mapear desflorestamento na Floresta Amazônica (Zalloti Júnior et. al., 2006).

Neste contexto, o objetivo deste trabalho foi avaliar o potencial do dado SAR adquirido pelo R99B no modo de imageamento polarimétrico em banda $\mathrm{L}$ para discriminar incremento de desflorestamento (área desflorestada por corte raso há no máximo um ano) na Amazônia. Para tanto, foram realizadas classificaçóes automáticas do dado SAR de uma área teste localizada no Estado do Acre. Os resultados das classificaçôes foram avaliados a partir da análise comparativa com o mapa de desflorestamento produzido no projeto PRODES do INPE.

\section{MATERIAL E MÉTODOS}

\section{ÁREA TESTE}

A área teste localiza-se na região leste do Estado do Acre, onde se estende por $1053 \mathrm{~km}^{2}$ entre os municípios de Rio Branco e Bujari (Figura 1). A vegetação predominante é a Floresta Ombrófila Aberta Submontana com presença de bambus e palmeiras. Os tipos de solo mais representativos são os Argissolos e os Gleissolos. A altitude média é de aproximadamente $200 \mathrm{~m}$. Na maior parte da área, o relevo é dissecado de topo tabular, com densidade de drenagem média e baixo grau de entalhamento dos canais (RADAM, 1978).

A ocupação desta área se concentra ao longo da rodovia estadual AC-090 e de suas ramificaçóes. Ao longo destas estradas há assentamentos rurais, pólos agroflorestais e áreas de manejo, onde as principais atividades são o extrativismo vegetal (castanha, exploração de madeira em tora e para lenha; e látex), a agricultura familiar (produçáo de arroz, feijáo, mandioca e milho) e a pecuária extensiva. Nas proximidades do Rio do Rola (porção sul) há populações ribeirinhas tradicionais (ACRE, 2000).

\section{BASE DE DADOS}

Neste trabalho foram utilizadas duas imagens SAR multipolarizadas adquiridas em banda L pelo sistema aerotransportado R99B no dia 31 de julho de 2006. Estas imagens correspondem aos segmentos 7 e 8 da missão de imageamento solicitada pelo SIPAM (Sistema de Proteção da Amazônia) que recobriu o Estado do Acre entre os meses de julho e agosto de 2006. As características dos dados SAR utilizados são apresentadas na Tabela 1. 


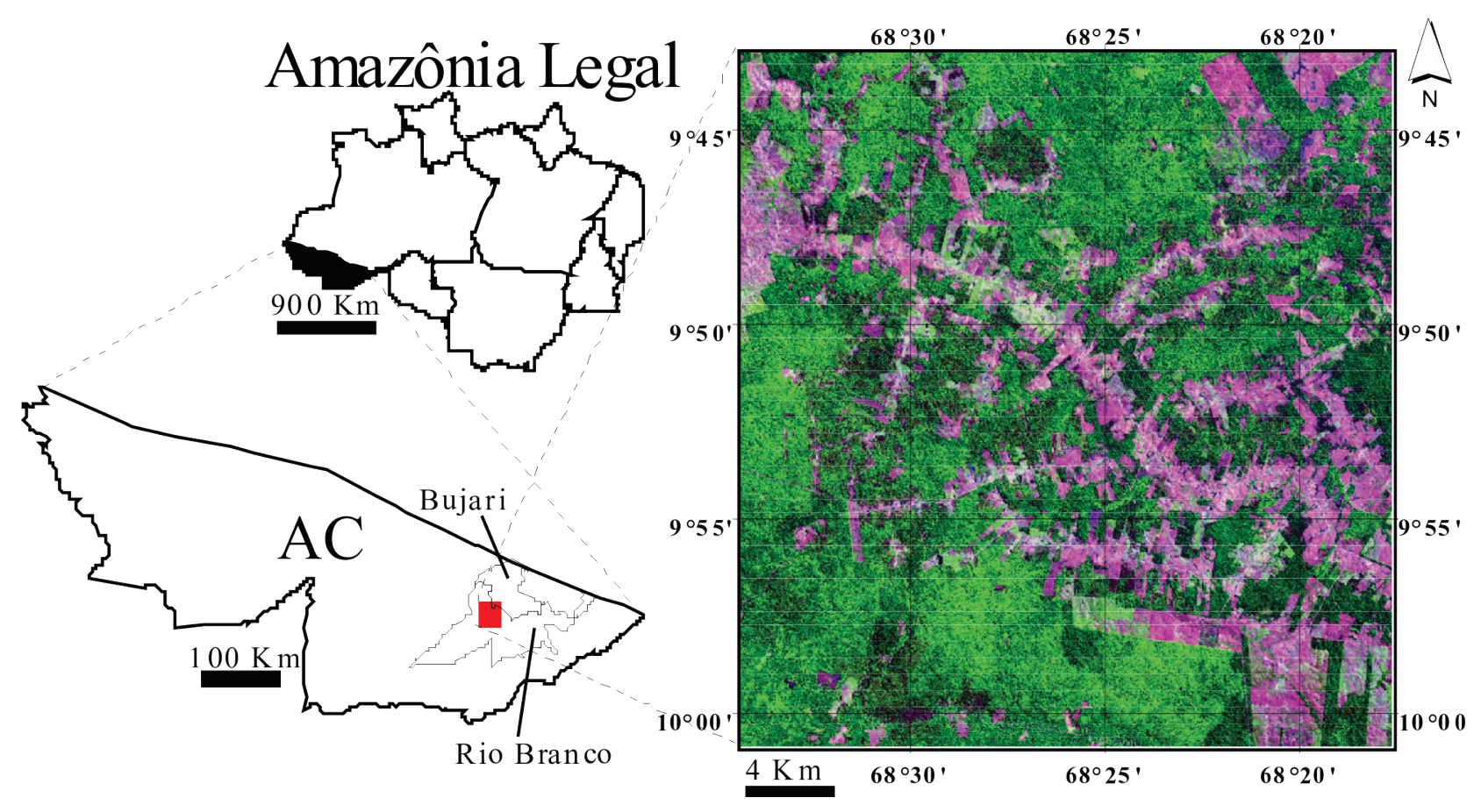

Figura 1 - Localização da área teste. Imagem Landsat TM (5R4G3B) de 13 de Agosto de 2006.

Tabela 1 - Características dos dados SAR.

\begin{tabular}{ll}
\hline Modo de imageamento & Quad L \\
\hline Freqüência central de operação & $\begin{array}{l}1,28 \mathrm{GHz} \\
\text { (banda L) }\end{array}$ \\
Polarizações & $\mathrm{HH}, \mathrm{HV} \mathrm{e} \mathrm{VV}$ \\
Resolução & $4 \mathrm{~m}$ (azimute) X \\
$6 \mathrm{~m}$ (alcance)
\end{tabular}

A imagem ETM+ Geocover/Landsat de 2 de agosto de 1999 (órbita 002/ponto 67) foi utilizada no georreferenciamento do dado SAR. As imagens TM/Landsat de 11 de setembro de 2005 (órbita 002/ponto 67), 28 de julho de 2006 (órbita 002/ponto 67) e 13 de agosto de 2006 (órbita 002/ponto 67) auxiliaram as amostragens e análises realizadas. $\mathrm{O}$ mapa de desflorestamento de 2006 (referente à cena TM/Landsat 002/67) produzido no projeto PRODES foi adotado como dado de referência (Figura 2).

\section{METODOLOGIA}

Os dados SAR dos segmentos 7 e 8 da Missão Acre foram submetidos à um procedimento de reamostragem por média espacial (multilook) nas

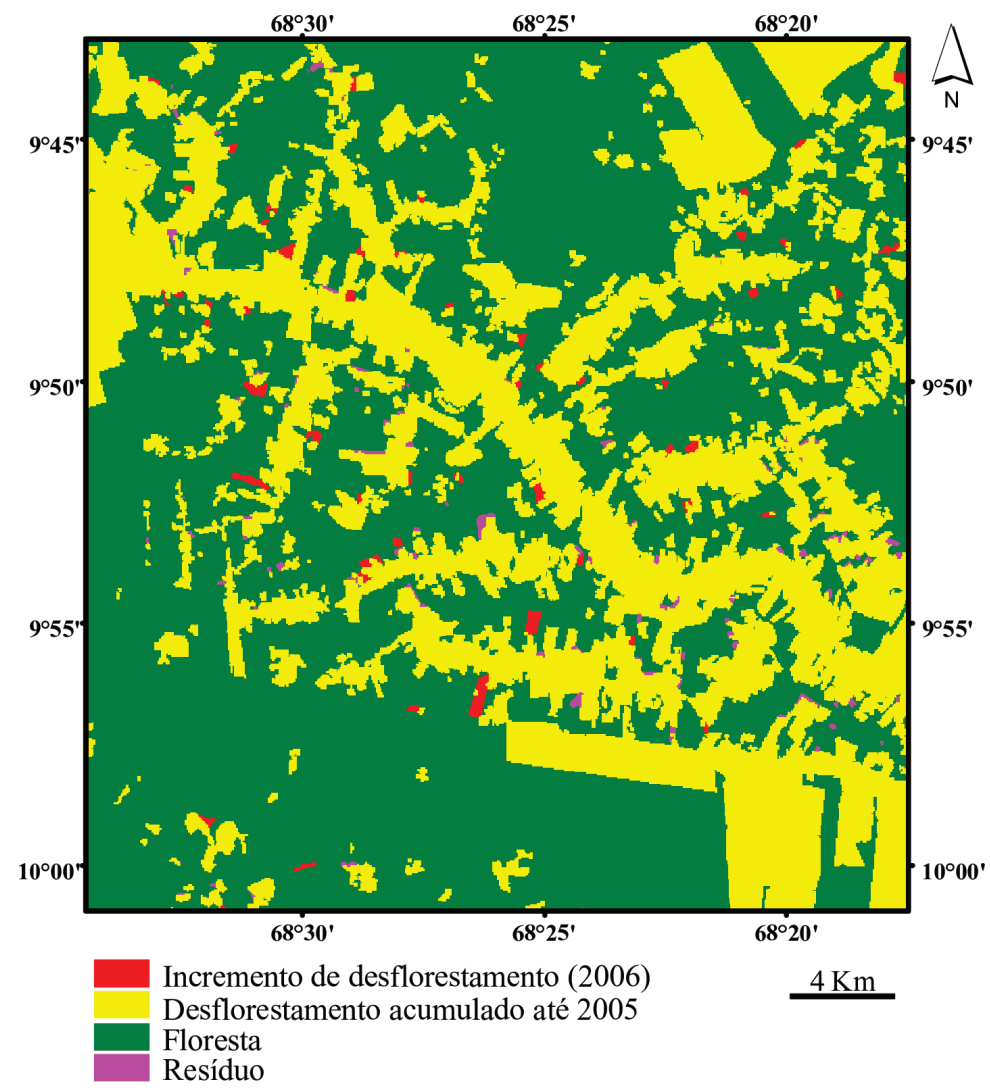

Figura 2 - Mapa de desflorestamento da área teste produzido pelo PRODES/2006. A classe resíduo corresponde a erros de omissão do mapeamento do PRODES. A classe incremento de desflorestamento foi mapeada com a imagem Landsat TM de 13 de Agosto de 2006. 
direçóes de alcance e azimute (6x6), o que produziu imagens cuja dimensão do pixel é $30 \mathrm{~m}$. Este procedimento foi empregado para reduzir o ruído speckle (Oliver e Quegan, 1998) e facilitar a realização das amostragens a partir do mapa produzido no projeto PRODES e das análises de concordância entre este mapa e os dados temáticos resultantes das classificaçóes da imagem SAR. Em seguida as imagens SAR foram registradas em relação à imagem ETM+ Geocover/Landsat (002/67). Após o registro, um mosaico que cobre a área teste foi construído com as imagens SAR dos segmentos 7 e 8.

O potencial do dado SAR para discriminar incremento de desflorestamento foi avaliado a partir da aplicação do classificador MAXVER (Máxima Verossimilhança), seguido do algoritmo ICM (Iterated Conditional Modes), o qual consiste em uma técnica de classificação contextual baseada na teoria de campos Markovianos (Vieira, 1996). As classificaçôes foram realizadas com uma $(\mathrm{HH}, \mathrm{HV}$ eVV), duas $(\mathrm{HH}+\mathrm{VV}, \mathrm{HH}+\mathrm{HV}$ e $\mathrm{HV}+\mathrm{VV})$ e três polarizaçôes $(\mathrm{HH}+\mathrm{HV}+\mathrm{VV})$ em amplitude.

A distribuição normal foi utilizada em todas as classificaçóes. Neste caso, a suposição de normalidade é aceitável, pois com a elevação do número equivalente de looks (NEL) após a aplicação da média espacial $(\mathrm{NEL}=17,2)$, a distribuição

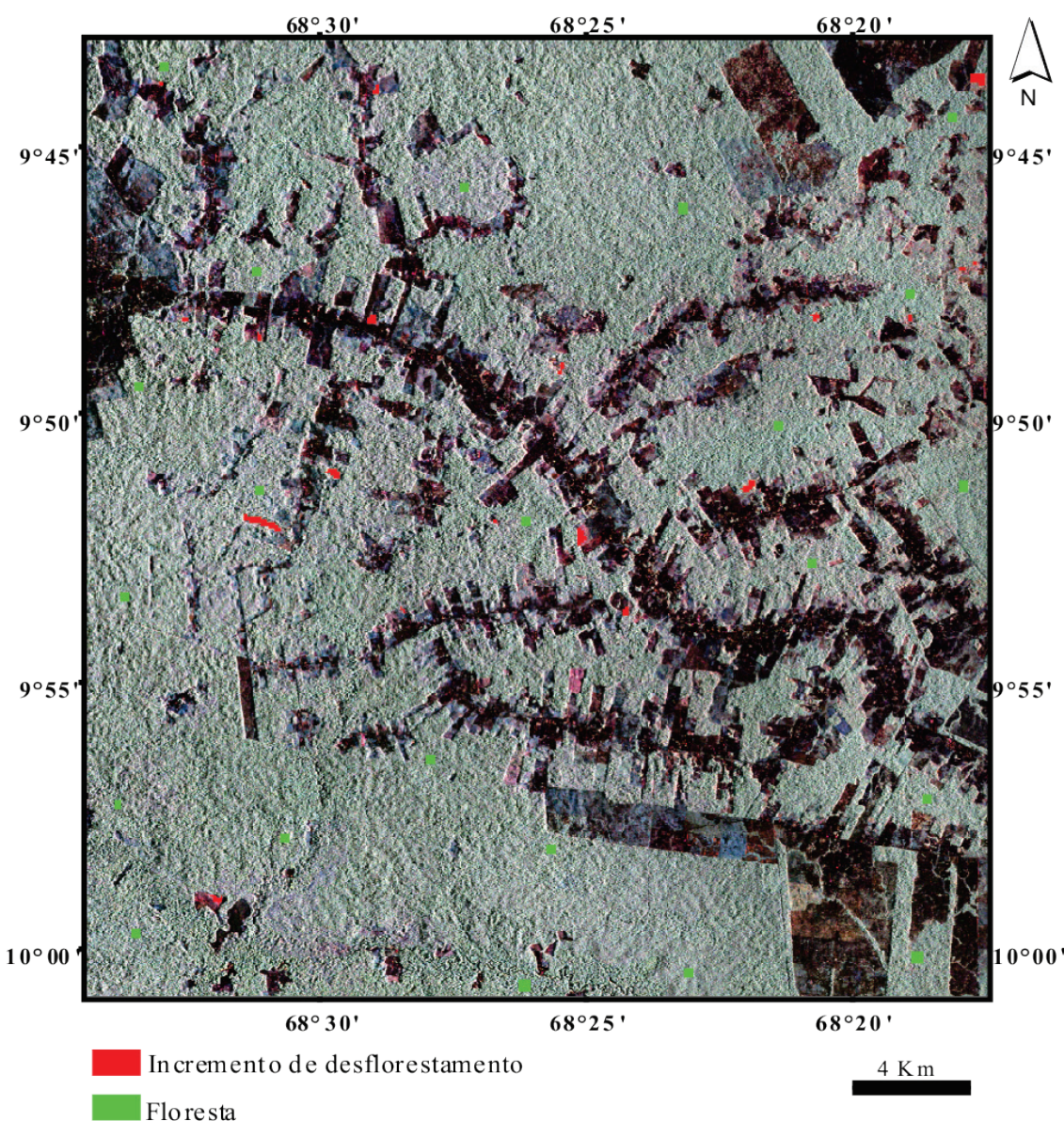

$\Gamma^{1 / 2}(n, n)$ da imagem SAR em amplitude tende a se aproximar de uma distribuição Gaussiana (Oliver e Quegan, 1998).

Neste trabalho, as classes de interesse foram definidas como floresta e incremento de desflorestamento. As amostras de treinamento das classes floresta e incremento de desflorestamento utilizadas na classificação foram obtidas a partir do mapa do PRODES de 2006 (Figura 2), com o auxílio da imagem TM/Landsat de 13 de agosto de 2006. Este mapa foi submetido à aplicação de um filtro morfológico de erosão (5x5), o que eliminou os pixels próximos às bordas das classes para assegurar a fidelidade das amostras.

De posse do mapa erodido, foram coletadas 1427 amostras (pixels)/19 polígonos de treinamento da classe incremento de desflorestamento e 1876 amostras (pixels)/21 polígonos da classe floresta. A Figura 3 mostra os polígonos que contêm as amostras (pixels) de treinamento de floresta (polígonos verdes) e de incremento de desflorestamento (polígonos vermelhos) sobre o mosaico de imagens SAR. Os desflorestamentos acumulados até 2005 foram desconsiderados a partir da aplicação de uma máscara obtida do mapa produzido no projeto PRODES. A máscara é composta pelas classes "desflorestamento acumulado" e "resíduo".

A avaliação dos resultados das classificaçóes foi realizada a partir da análise de concordância com o mapa do PRODES erodido. A análise de concordância consistiu na construção e comparação das matrizes de confusão e no cálculo das estatísticas derivadas dessas matrizes (índice kappa, erros de omissão e de inclusão e acurácias do usuário - razão entre o número de amostras de referência da classe "c" corretamente classificadas e o total de amostras de referência classificadas na classe "c" - e do produtor - razão entre o número de amostras de referência da classe " $\mathrm{c}$ " corretamente classificadas e o número total de amostras de referência da classe "c") (Congalton e Green, 1999). O mapa erodido foi utilizado
Figura 3 - Mosaico SAR R99B (RHH-GHV-BVV) da área teste. Os polígonos vermelhos e verdes contêm as amostras de treinamento das classes incremento de desflorestamento e floresta, respectivamente. 


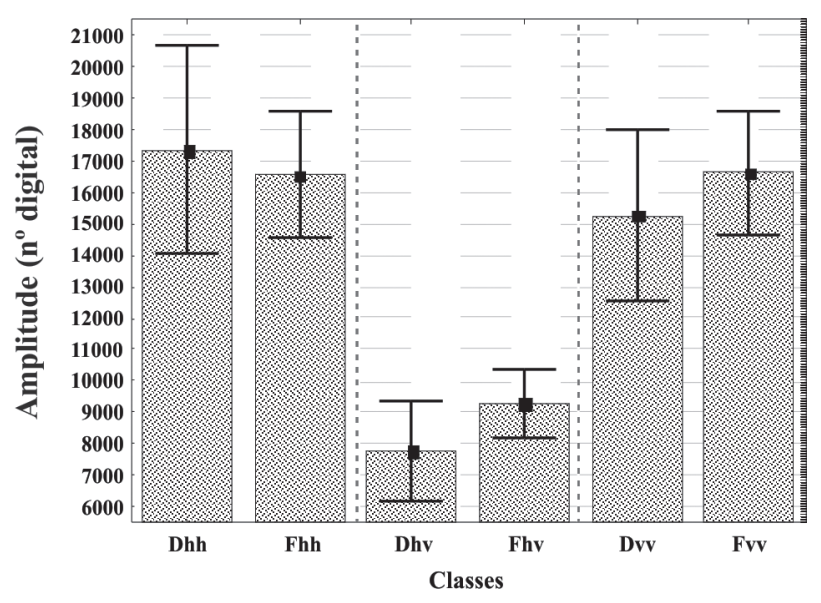

Figura 4 - Amplitudes médias (barras) e desvios dos conjuntos de amostras de treinamento de incremento de desflorestamento (D) e floresta (F) nas polarizações HH, HV e VV.

com o objetivo de reduzir o efeito dos erros de co-registro nas análises de concordância.

\section{RESULTADOS E DISCUSSÃO}

As médias e os desvios das amplitudes dos conjuntos de amostras de treinamento de floresta e incremento de desflorestamento são apresentados na Figura 4 com o objetivo de analisar o comportamento destas classes. A dispersão da amplitude da classe incremento de desflorestamento é superior a da floresta nas três polarizaçóes, o que pode indicar a maior heterogeneidade desta classe em função da existência de áreas em diferentes estágios de desflorestamento por corte raso. $\mathrm{O}$ teste $t$-student apontou que a amplitude média do conjunto de amostras de floresta é superior a do conjunto de amostras de incremento de desflorestamento nos canais $\mathrm{HV}$ e VV, a um nível de significância de $5 \%$ (p-valor $=0,064)$. Na polarização $\mathrm{HH}$, a resposta média dos incrementos de desflorestamento é maior que a da floresta, o que corrobora com o apresentado por Rignot et al. (1997). Segundo estes autores, o elevado retorno em banda L e polarização $\mathrm{HH}$ ocorre em áreas recém desflorestadas devido à interação com troncos e galhos que permanecem derrubados sobre o solo logo após os estágios de corte e queima da floresta.

O teste Komolgorov-Smirnov (nível significância de 5\%) determinou que as amostras de treinamento de floresta e incremento de desflorestamento são ajustadas por uma distribuição normal nas três polarizaçóes em amplitude (o menor p-valor obtido no teste Komolgorov-Smirnov foi igual a 0,061, para as amostras da classe incremento de desflorestamento no canal HV). A Figura 5 apresenta as densidades (distribuiçóes normais) dos conjuntos de amostras de treinamento das classes incremento de desflorestamento e floresta. A sobreposição entre (a)

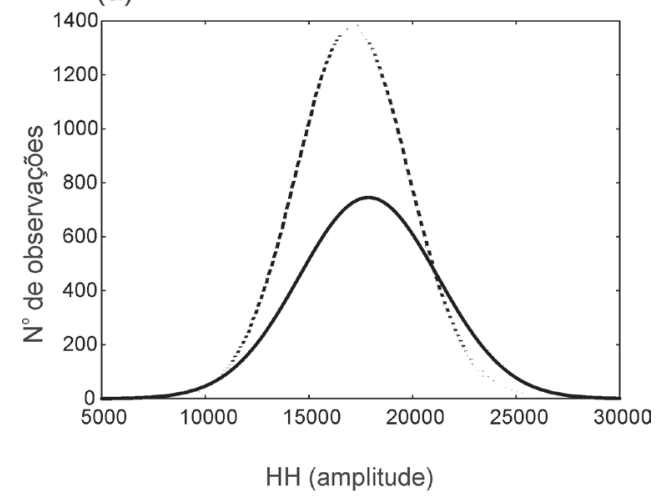

(b)

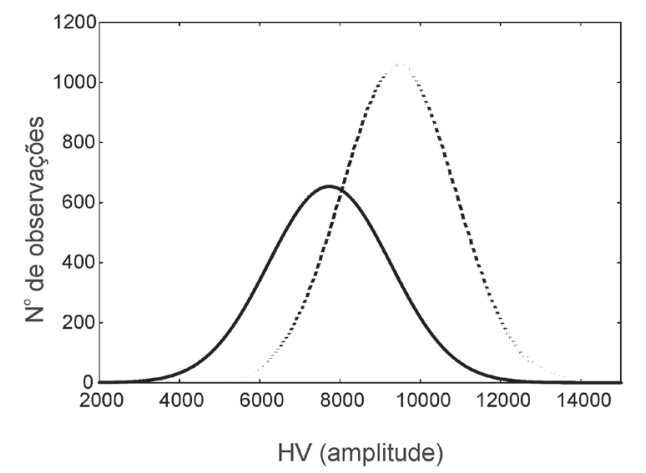

(c)

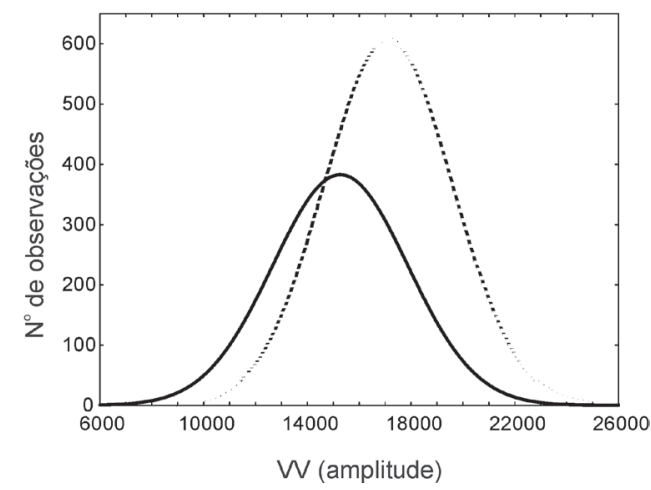

Figura 5 - Densidades dos conjuntos de amostras das classes floresta (linha tracejada) e incremento de desflorestamento (linha cheia) para as polarizações. (a) Densidade dos conjuntos de amostras na polarização $\mathrm{HH}$; (b) densidade dos conjuntos de amostras na polarização HV e (c) densidade dos conjuntos de amostras na polarização VV.

as densidades das classes é quase total nas polarizaçóes $\mathrm{HH} \mathrm{e} \mathrm{VV}$ e um pouco menor na polarização HV. A melhor separação das classes em HV se deve ao contraste criado pela sensibilidade da polarização cruzada ao espalhamento volumétrico, produzido no interior das copas das árvores, e pela baixa resposta de solos expostos ou cobertos por vegetação baixa (Saatchi et al., 1997). A grande sobreposição entre as densidades indica que pode ser 
(A)

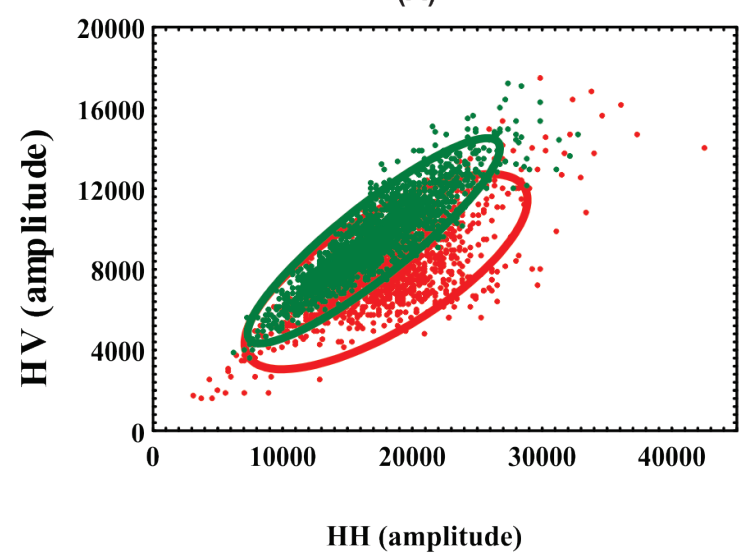

(C)

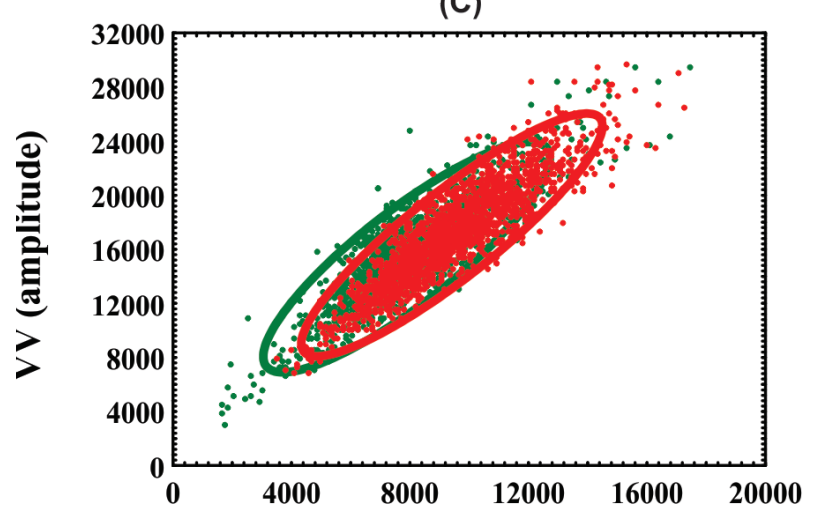

HV (amplitude)

(B)

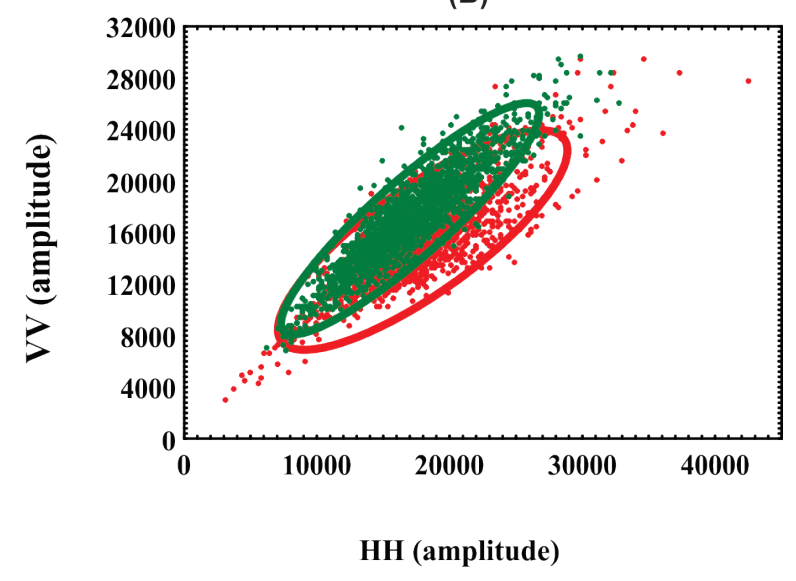

Figura 6 - Diagramas de dispersão das amostras das classes floresta (pontos verdes) e incremento de desflorestamento (pontos vermelhos). (a) Par de polarizações $\mathrm{HH}+\mathrm{HV}$; (b) par de polarizações $\mathrm{HH}+\mathrm{VV}$; (c) par de polarizações HV+VV. As elipses representam distribuições normais bivariadas (95\%) das amostras de floresta (elipse verde) e incremento de desflorestamento (elipse vermelha).
Tabela 2 - Valores de índice kappa obtidos para cada classificação realizada. Os valores de kappa apresentados em negrito e itálico correspondem a matrizes de confusão estatisticamente iguais a 5\% de significância.

\begin{tabular}{|c|c|c|}
\hline Classificação & kappa & $\sigma_{k}^{2}$ \\
\hline $\mathrm{HH}$ & 0,0671 & 0,0000059 \\
\hline HV & 0,0466 & 0,0000008 \\
\hline WV & 0,0073 & 0,0000000428 \\
\hline$H H+H V$ & 0,6884 & 0,0000288 \\
\hline $\mathrm{HH}+\mathrm{VV}$ & 0,5443 & 0,0000331 \\
\hline $\mathrm{HV}+\mathrm{VV}$ & 0,0582 & 0,0000010 \\
\hline$H H+H V+V V$ & 0,6906 & 0,0000292 \\
\hline
\end{tabular}

difícil separar as classes floresta e incremento de desflorestamento com a amplitude de apenas uma polarizaçáo.

Os diagramas de dispersão da Figura 6 mostram que o par de polarizaçóes $\mathrm{HH}+\mathrm{HV}$ parece ser o que melhor separa as classes de interesse, ainda que com considerável sobreposição entre as distribuiçôes bivariadas. No caso do par $\mathrm{HV}+\mathrm{VV}$, a sobreposição quase total entre as distribuiçóes indica que as classes floresta e incremento de desflorestamento podem ser praticamente indistinguíveis.

A Tabela 2 apresenta os valores de índice kappa de cada uma das classificaçôes realizadas. Quando somente um canal foi utilizado, o resultado obtido foi insatisfatório. Neste caso, o maior valor de kappa foi alcançado pela classificação realizada com HH ( $k=0,0671)$. Ao utilizar duas polarizaçôes, observouse um ganho de acurácia em relação às classificaçôes realizadas com somente um canal. O melhor resultado foi alcançado pelo par de polarizaçôes $\mathrm{HH}+\mathrm{HV}(\mathrm{k}=0,6884)$ e o pior, pelo par $\mathrm{HV}+\mathrm{VV}(\mathrm{k}=0,0582)$, o que corrobora com o apresentado nos diagramas de dispersão da Figura 6. Quando os três canais foram considerados, o valor de kappa foi de 0,6906. Estes resultados evidenciam a importância da multipolarização para discriminar incrementos de desflorestamento.

$\mathrm{O}$ teste $\mathrm{z}$ bilateral determinou que as matrizes de confusāo das classificaçôes realizadas com $\mathrm{HH}+\mathrm{HV}$ e $\mathrm{HH}+\mathrm{HV}+\mathrm{VV}$ são estatisticamente iguais, a $5 \%$ de significância ( $p$-valor $=0,058$ ), o que indica que a informação contida no par de polarizações $\mathrm{HH}+\mathrm{HV}$ é suficiente para discriminar a classe incremento de desflorestamento com boa acurácia. Portanto, o canal VV pode não oferecer informação adicional. Este resultado está de acordo com o apresentado por Hoekman e Quinónes (2000). Entretanto, difere do obtido em estudo realizado por Santos et al. (2008), o qual aponta o par de polarizaçóes HV+VV como o melhor para discriminar desflorestamentos.

$\mathrm{Na}$ classificação com $\mathrm{HH}$, as acurácias do produtor para as classes incremento de desflorestamento e floresta foram de 19,38\% (915/4722) e 97,09\% (587565/605204), respectivamente. Portanto, apesar do valor de kappa da classificação com $\mathrm{HH}$ ser o melhor dentre as classificaçôes com polarizaçóes individuais, 
Tabela 3 - Matriz de confusão da classificação realizada com a polarização HH. E.I. significa erros de inclusão; E.0. significa erros de omissão; A.U. significa acurácia do usuário e A.P. significa acurácia do produtor.

\begin{tabular}{lccccc}
\hline Classes & $\begin{array}{c}\text { Incremento } \\
\text { desflorestamento }\end{array}$ & Floresta & Total & $\begin{array}{c}\text { E.I. } \\
(\%)\end{array}$ & $\begin{array}{c}\text { A.U. } \\
(\%)\end{array}$ \\
\hline $\begin{array}{l}\text { Incremento } \\
\text { desflorestamento }\end{array}$ & 915 & 17639 & 18554 & 95,07 & 4,63 \\
Floresta & 3807 & 587565 & 591372 & 0,64 & 99,36 \\
Total & 4722 & 605204 & 609928 & & \\
E.0. (\%) & 80,62 & 2,91 & & & Kappa \\
A.P. (\%) & 19,38 & 97,09 & & & 0,0671 \\
\hline
\end{tabular}

Tabela 4 - Matriz de confusão da classificação realizada com a polarização HV. E.I. significa erros de inclusão; E.0. significa erros de omissão; A.U. significa acurácia do usuário e A.P. significa acurácia do produtor.

\begin{tabular}{lccccc}
\hline Classes & $\begin{array}{c}\text { Incremento } \\
\text { desflorestamento }\end{array}$ & Floresta & Total & $\begin{array}{c}\text { E.I. } \\
(\%)\end{array}$ & $\begin{array}{c}\text { A.U. } \\
(\%)\end{array}$ \\
\hline $\begin{array}{l}\text { Incremento } \\
\text { desflorestamento }\end{array}$ & 3094 & 94104 & 97198 & 96,82 & 3,18 \\
Floresta & 1628 & 511100 & 512728 & 0,32 & 99,68 \\
Total & 4722 & 605204 & 609928 & & \\
E.O. (\%) & 34,48 & 15,55 & & & Kappa \\
A.P. (\%) & 65,52 & 84,45 & & & 0,0466 \\
\hline
\end{tabular}

os erros de omissão para a classe incremento de desflorestamento foram muito altos $(80,62 \%, 3807 / 4722)$ (Tabela 3). Isto indica que o dado na polarizaçáo $\mathrm{HH}$ possui limitaçôes para detectar incremento de desflorestamento, o que corrobora com o apresentado por Bufalo (2005).

Nas classificaçóes realizadas com o canal HV e com o par de polarizaçóes $\mathrm{HV}+\mathrm{VV}$, as acurácias do produtor para as classes incremento de desflorestamento e floresta foram de $65,52 \%$ (3094/4722) e $84,45 \%$ (511100/605204), e $72,91 \%$

Tabela 5 - Matriz de confusão da classificação realizada com par de polarizações HH+HV. E.I. significa erros de inclusão; E.0. significa erros de omissão; A.U. significa acurácia do usuário e A.P. significa acurácia do produtor.

\begin{tabular}{lccccc}
\hline Classes & $\begin{array}{c}\text { Incremento } \\
\text { desflorestamento }\end{array}$ & Floresta & Total & E.I. (\%) & A.U. (\%) \\
\hline $\begin{array}{l}\text { Incremento } \\
\text { desflorestamento }\end{array}$ & 3396 & 1712 & 5108 & $33,52 \%$ & $66,48 \%$ \\
Floresta & 1326 & 603492 & 604818 & $0,22 \%$ & $99,78 \%$ \\
Total & 4722 & 605204 & 609926 & \\
E.0. (\%) & $28.08 \%$ & $0.28 \%$ & & & Kappa \\
A.P. (\%) & $71,92 \%$ & $99,72 \%$ & & 0,6884 \\
\hline
\end{tabular}

(3443/4722) e 85,56\% (517788/605204), respectivamente. Porém, apesar de terem discriminado a maior parte dos incrementos de desflorestamento, as classificaçôes com os canais HV (erros de inclusão 96,82\%, 94104/97198) e $\mathrm{HV}+\mathrm{VV}$ (erros de inclusão 96,21\%, 87416/90859) foram insatisfatórias devido aos altos erros de inclusão para esta

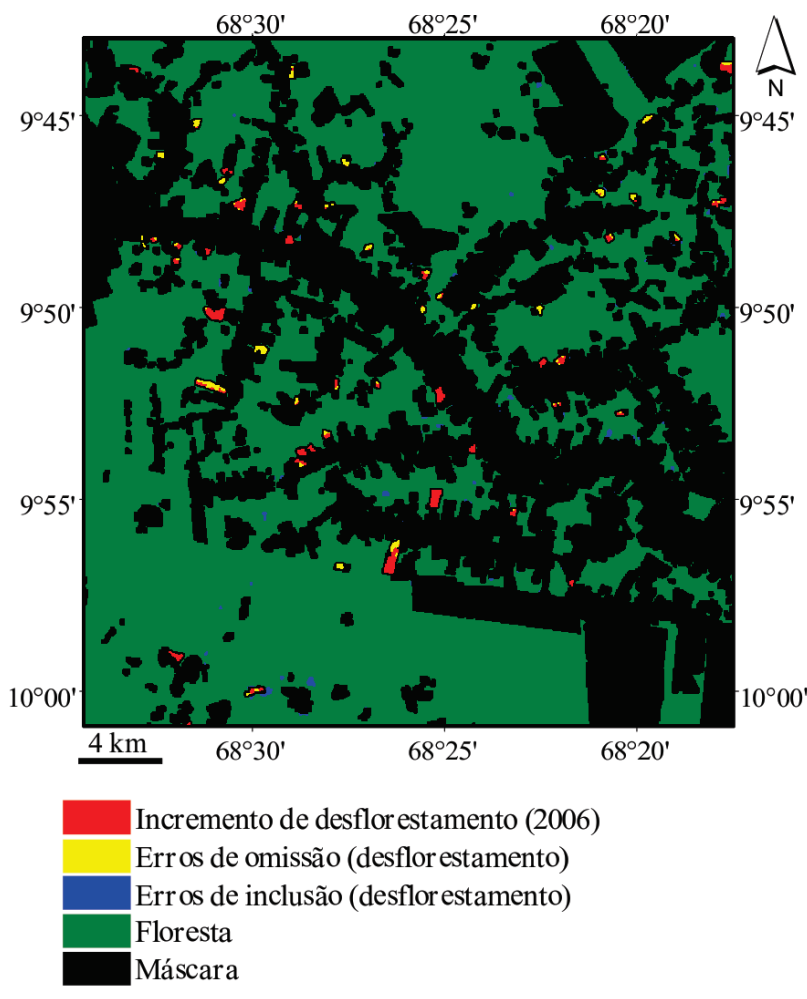

Figura 7 - Espacialização da matriz de confusão da classificação MAXVERICM realizada com o par de polarizações $\mathrm{HH}+\mathrm{HV}$.

classe. A Tabela 4 mostra a matriz de confusão da classificação realizada com a polarização $\mathrm{HV}$.

$\mathrm{O}$ resultado da classificação com o par $\mathrm{HH}+\mathrm{HV}$, a qual obteve a melhor concordância com o mapa do PRODES, é apresentado na Tabela 5 e na Figura 7. Neste caso, $71,92 \%$ das amostras de referência da classe incremento de desflorestamento e $99,72 \%$ das amostras de floresta foram corretamente classificadas. Os erros de inclusão foram predominantes para a classe incremento de desflorestamento $(33,52 \%)$. Os erros de omissáo representam $28.08 \%$ das amostras de referência de incremento de desflorestamento.

A inspeção visual do resultado da classificação MAXVERICM do par de polarizaçôes $\mathrm{HH}+\mathrm{HV}$ foi realizada com o auxílio das imagens TM/Landsat. Esta análise mostrou que grande parte dos incrementos de desflorestamento corretamente classificados correspondem a regiôes onde há ocorrência significativa de solo exposto. Alguns dos erros de inclusão (regiôes azuis no mapa da Figura 7) da classe incremento de desflorestsmento observados nesta inspeção se devem a erros de omissão do mapa do PRODES (Figura 8a), fato que prejudicou a avaliação dos resultados das classificaçôes.

Grande parte dos erros de omissão da classe incremento de desflorestamento corresponde a áreas queimadas e áreas onde o 


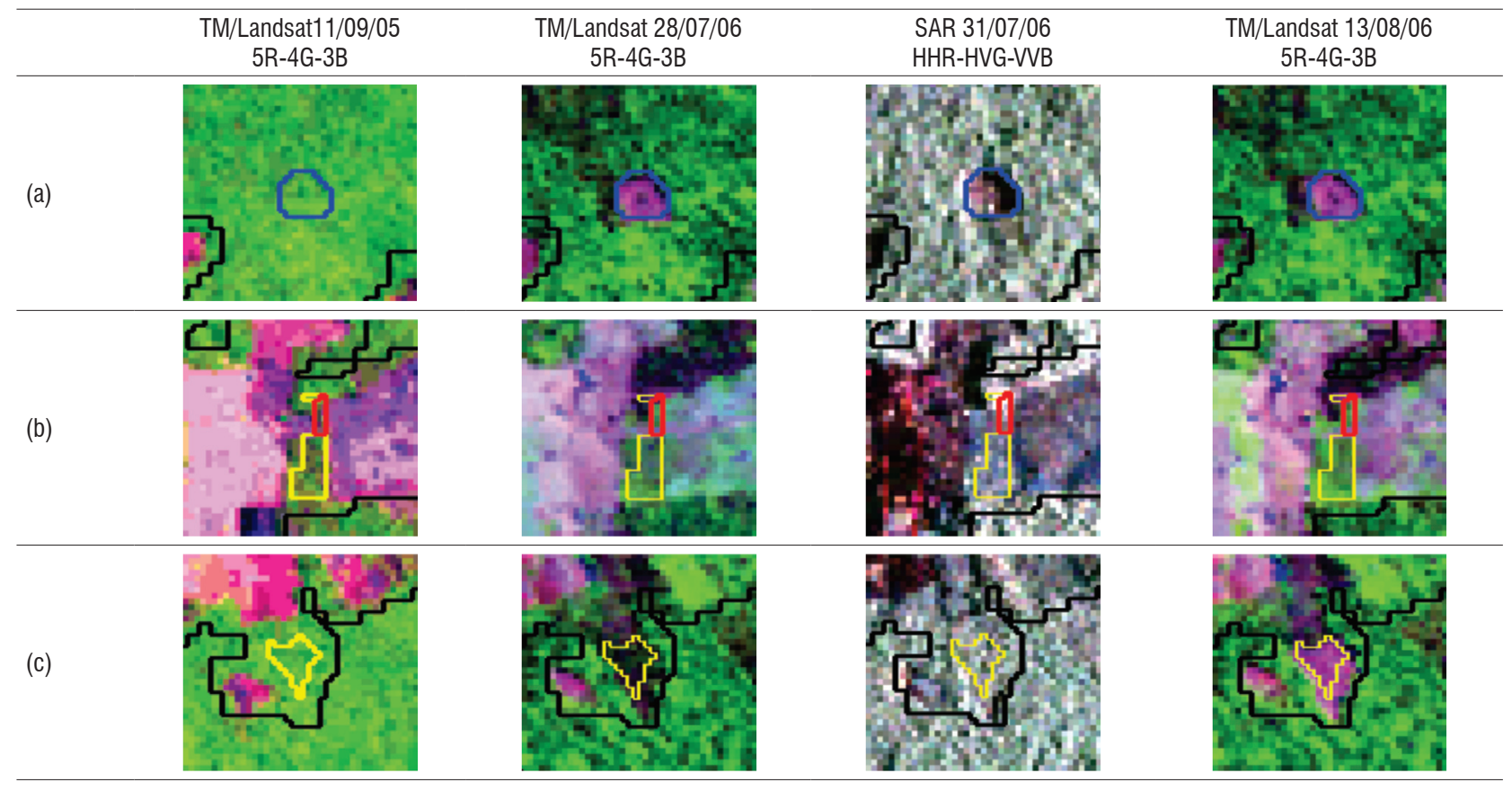

Figura 8 - Erros de omissão (polígonos amarelos) e de inclusão (polígono azul) para a classe incremento de desflorestamento da classificação MAXVER-ICM realizada com HH+HV. 0 polígono vermelho corresponde a uma área de incremento de desflorestamento corretamente classificada.

processo de regeneração da vegetação já foi iniciado. O erro de omissão da classe incremento de desflorestamento (polígono amarelo) apresentado na da Figura 8b corresponde a uma área coberta por capoeira que apresenta um comportamento semelhante ao da floresta na imagem SAR, porém com retorno menos intenso nos canais HH e VV. De acordo com Rignot et al. (1997), a vegetação que cresce durante um ano após o corte e queima da floresta pode ser capaz de mascarar a resposta do solo e provocar a diminuição do retorno em banda $\mathrm{L}$ e polarização $\mathrm{HH}$, o que reduz o contraste entre a floresta e os desflorestamentos. Dutra et al. (2007) relataram que uma área em processo de regeneração há um ano não pôde ser visualmente identificada em uma imagem multipolarizada ALOS/PALSAR da regiāo da Floresta Nacional de Tapajós $-\mathrm{PA}$.

$\mathrm{O}$ incremento de desflorestamento omitido (polígono amarelo) da Figura 8c consiste em uma feição de queimada (observada na imagem TM/Landsat do dia 28/07/2006) que evoluiu para uma área onde há ocorrência significativa de solo exposto (observada na imagem do dia 13/08/2006) no intervalo de 16 dias. Na imagem SAR não é possível observar variaçốes entre os comportamentos da floresta e o da área desflorestada. No momento da aquisição do dado SAR, esta área possivelmente se encontrava em um estágio inicial do processo de desflorestamento e provavelmente possuía galhos e troncos derrubados sobre o solo, o que pode dificultar sua discriminação (Almeida Filho et al., 2005).

\section{CONCLUSÃO}

O potencial de discriminação de incremento de desflorestamento do dado SAR adquirido em banda L pelo sistema aerotransportado R99B foi avaliado neste trabalho a partir de classificaçóes MAXVER e ICM com uma, duas e três polarizaçóes. Os resultados mostraram que o dado SAR multipolarizado em banda L possui bom potencial para discriminar incremento de desflorestamento na Amazônia. Além disso, o fato da classificação realizada com o par $\mathrm{HH}+\mathrm{HV}$ ter obtido a melhor concordância com o mapa do PRODES pode justificar a configuraçáo do modo de imageamento operacional do sensor orbital ALOS/PALSAR $(\mathrm{HH}+\mathrm{HV})$ para aplicaçóes em floresta tropical.

\section{BIBLIOGRAFIA CITADA}

Acre, Governo Do Estado Do Acre. 2000. State of Acre ecologicaleconomic zoning - socioeconomic and territorial occupation. Secretaria de Estado de Planejamento e Coordenação, Programa Estadual de Zoneamento Ecológico-Econômico, Rio Branco, v. 2, $156 \mathrm{p}$ (in Portuguese).

Almeida Filho, R.; Rosenqvist, A.; Shimabukuro, Y. E.; Dos Santos, J. R. 2005. Evaluation and perspectives of using multitemporal L-band SAR data to monitor deforestation in the Brazilian Amazonia. IEEE Geoscience and Remote Sensing Letters, 2(4): 409-412.

Asner, G. P. 2001. Cloud cover in Landsat observations of the Brazilian Amazon. International Journal of Remote Sensing, 22(18): 3855-3862. 
Bufalo, A. C. 2005. Assessing Rain Forest Mapping Mission radar mosaics data to deforestation monitoring in Brazilian Amazon. Dissertação de Mestrado, Instituto Nacional de Pesquisas Espaciais (INPE), São José dos Campos, São Paulo. 169 pp (in Portuguese).

Câmara, G.; Valeriano, D. D. M.; Soares, J. V. 2006. Methodology for calculation of Amazon deforestation annual rate. São José dos Campos: Instituto Nacional de Pesquisas Espaciais (INPE), $22 \mathrm{p}$ (in Portuguese).

Congalton, R.G.; Green, K. G. 1999. Assessing the accuracy of remotely senseddata: principles and practices. New York: Boca Raton, FL, 137p

Dutra, L. V.; Scofield, G.; Aboud, S. R. N.; Negri, R. G.; Freitas, C. C.; Mura, J. C.; Sant'anna, S.; Gama, F. F. 2007. ALOS/ PALSAR polarimetric data for land use/land cover classification in Amazon. In: The First Joint PI Symposium of ALOS Data Nodes for ALOS Science Program, Kyoto, Proceedings...JAXA: Kyoto, 2007.

Freitas, C. C.; Soler, L. S.; Sant'anna, S. J.S.; Dutra, L. V.; Santos, J. R.; Mura, J. C.; Correia, A. H. 2008. Land use and land cover mapping in the Brazilian Amazon using airborne P-band SAR data. IEEE Transactions on Geoscience and Remote Sensing.v. 46, n. 10.

Hoekman, D. H.; Quiñones, M. J. 2000. Land cover type and biomass classification using AirSAR data for evaluation of monitoring scenarios in the Colombian Amazon. IEEE Transactions on Geoscience and Remote Sensing, 38(2): 685696.

Instituto Nacional De Pesquisas Espaciais (INPE). 2008. Amazon Raionforest satellite monitoring. São José dos Campos-SP: Instituto Nacional de Pesquisas Espaciais (INPE), 143p (in Portuguese).

Kuplich, T. M. 2006. Classifying regenerating forest stages in Amazônia using remotely sensed images and a neural network. Forest Ecology and Management, The Netherlands, v. 234, p. $1-9$.

Oliver, C.; Quegan, S. 1998. Understanding synthetic aperture radar images. Norwood, MA: Artech House, 478 p.
RADAM Brasil. 1978. Sheet SC.19 Rio Branco: geology, geomorfology, pedology, vegetatio and land use potential. Ministério das Minas e Energia, Departamento Nacional de Produção Mineral (DNPM), Levantamento de Recursos Naturais, Rio de Janeiro, v. 17, 554 p (in Portuguese).

Rignot, E.; Salas, W. A.; Skole, D. L. 1997. Mapping deforestation and secondary growth in Rondônia, Brazil, using imaging radar and thematic mapper data. Remote Sensing of Environment, 59(2): 167-179.

Saatchi, S. S.; Soares, J. V.; Alves, D. S. 1997. Mapping deforestation and land use in Amazon rainforest by using SIR-C imagery. Remote Sensing of Environment, (59)2: 191-202.

Santos, J. R.; Mura, J. C.; Paradella, W. R.; Dutra, L. V.; Gonçalves, F. G. 2008. Mapping recent deforestation in the Brazilian Amazon using simulated L-band MAPSAR images. International Journal of Remote Sensing, v. 29, n. 16, p. 4879-4884.

Santos, J. R.; Freitas, C. C.; Araujo, L. S.; Dutra, L. V.; Mura, J. C.; Gama, F. F.; Soler, L. S.; Sant'anna, S. J. S. 2003. Airborne P-band SAR applied to the aboveground biomass studies in the Brazilian tropical rainforest. Remote Sensing of Environment, 87(4): 482-493.

Sgrenzaroli, M.; Baraldi, A.; De Grandi, G. D.; Eva, H.; Achard, F. 2004. A novel approach to the classification of regionalscale Radar mosaics for tropical vegetation mapping. IEEE Transactions on Geoscience and Remote Sensing, 42(11): 2654-2669.

Vieira, P. R. 1996. Development of maximum likelihood classifiers and ICM for SAR images. Dissertação de Mestrado, Instituto Nacional de Pesquisas Espaciais (INPE), São José dos Campos, São Paulo. 251 pp (in Portuguese).

Zalotti, O. D. J.; Gonçalves, F. G.; Freitas, C. C.; Sant'anna, S. J. S.; Santos, J. R. 2006. Evaluating the potential of SAR-R99B L and $\mathrm{X}$ bands data for Amazon deforestation increment mapping. In: IEEE International Geoscience and Remote Sensing Symposium, 2006, Denver, Colorado. Proceedings...Piscataway: IEEE: 2662-2665.

Recebido em 20/11/2008

Aceito em 09/11/2009 